\title{
Editorial Vol. 10
}

\author{
Oliver Baumann ${ }^{1} \cdot$ John Joseph ${ }^{2} \cdot$ Metin Sengul $^{3} \cdot$ Brian $\mathrm{Wu}^{4}$
}

Published online: 12 April 2021

(c) The Author(s), under exclusive licence to Organizational Design Community 2021

The publication of the Journal of Organization Design's (JOD's) first issue in 2021 gives occasion for looking back and ahead. At the end of 2020, the terms of co-editors John Joseph (University of California, Irvine) and Metin Sengul (Boston College) ended.

\section{From the outgoing editors}

When we took over from the founding editors three years ago, we established five key goals for the journal:

1. Continue $J O D$ 's tradition of offering a variety of formats reflecting diversity of disciplines, theories, and methodologies in the field of organization design.

2. Expand the editorial team by appointing new associate editors and editorial board members, with a continued effort to give voice to scholarship from a variety of fields and ensure high-quality and timely feedback.

3. Grow JOD's efforts to produce special issues (collections) on fundamental theories and emerging phenomena.

\footnotetext{
Oliver Baumann

oliv@sam.sdu.dk

John Joseph

johnj2@uci.edu

Metin Sengul

metin.sengul@bc.edu

Brian Wu

wux@umich.edu
}

1 Department of Marketing \& Management, University of Southern Denmark, Campusvej 55, 5230 Odense M, Denmark

2 Paul Merage School of Business, University of California Irvine, Irvine, CA 92697, USA

3 Carroll School of Management, Boston College, 140 Commonwealth Avenue, Chestnut Hill, MA 02467, USA

4 Ross School of Business, University of Michigan, 701 Tappan St., Ann Arbor, MI 48109, USA
4. Increase $J O D$ 's impact, aiming to get approval for an impact factor from one of the major third-party institutions.

5. Increase social media presence to provide better visibility to $J O D$ articles and authors.

Thanks to a wonderful team of associate editors, editorial board members, Springer Nature associates, as well as guiding support from the Organizational Design Community (ODC), we were able to achieve them all. We saw the diversity of our board members expand and the variety of article formats continue to grow. In 2020, JOD published 24 articles, including 12 research papers, four points of view, four commentaries, one case study, one organization zoo, one research primer, and one translational. We were fortunate to have worked with so many great authors and guest editors across four special collections. With the help of Springer Nature, we expanded our visibility and witnessed a significant increase in JOD's social media presence. In the five years spanning 2016 (the year JOD moved to Springer Nature) through 2020, the journal's articles were accessed 419,813 times, with usage rising sharply each year to more than 197,000 in 2020 alone. We are especially thrilled that the journal was indexed by Scopus in April 2020, as the first outcome of our applications to selected abstracting and indexing services.

Serving as co-editors of $J O D$ has been a career highlight for us. Working with and learning from our organization design colleagues from across the globe has been extremely rewarding. It has been a pleasure to have played a part in moving $J O D$ and the field forward, and we look forward to staying involved. The journal is in good hands with Oliver and Brian taking the helm and we wish the new co-editors all the best. $J O D$ will remain a force that not only contributes to the current conversation in organization design, but also shapes the future of the field.

Beginning in January 2021, JOD's new co-editors are Oliver Baumann (University of Southern Denmark) and Brian Wu (University of Michigan, Ann Arbor). 


\section{From the incoming editors}

The start of our term coincides with an important step in the development of JOD. As of 2021, JOD transitioned from a fully open access journal (financed entirely by the Organizational Design Community) to a "hybrid" journal. This means that $J O D$ is now a subscription-based journal in which some articles are published open access. Given the growth in the number of published articles, this transition was necessary to establish a sustainable economic basis for JOD's future development and growth.

The "new" JOD comes with a new journal website as well as a new submission system. Members of the Organizational Design Community (ODC) as well as most academic readers (through their institutional subscriptions) will have full access to the journal, and of course all prior articles remain openly accessible through the new website. In addition, the editors of $J O D$ can select a number of articles each year to be published as open access, while authors can choose to make their accepted papers openly accessible by paying an article processing charge. Finally, open access agreements between Springer Nature and various university systems (e.g., the University of California system and Germany's Projekt DEAL) provide the option for eligible corresponding authors from participating institutions to publish their papers as open access at no direct cost.

As the new editors, we are also excited to announce an expanded set of associate editors and editorial board members. The top-level scholars-both junior and seniorthat make up JOD's editorial team reflect the momentum, impact, and diversity of research on organization design in general, and of JOD in particular. At the same time, this community ensures that manuscripts will be handled by relevant experts along a broad spectrum of topics and methods.

While we are taking over a journal that is on a very promising trajectory, thanks to the tremendous efforts by our predecessors, we cannot afford to stand still. We will therefore continue the work of growing the number of submissions by raising awareness for the journal and its unique profile. We will continue to grow the community of readers and authors. At the end of the day, JOD is a journal by the community and for the community. We will initiate new special issues that help focus attention on important problems and approaches. At this point, for example, we have an open call for a special issue on "Organization Design of Resilience in the Face of Unanticipated Global Crises" (co-edited by Dr. Arie Lewin and Dr. Liisa Välikangas), and we are in the planning stage for another special issue on the topic of "Organizing for Good", which will deal with the value of organization design for broader societal purposes such as the United Nations (UN) Sustainable Development Goals (SDGs). We will continue to work with Springer Nature on our journey toward an impact factor. And we will further ramp up JOD's social media activities.

In closing, let us say a few words about our vision for $J O D$. We want $J O D$ to remain a journal that, above all, cares about ideas - ideas that are fresh, provocative, and forwardlooking; we invite work that dares to be different while adhering to high scientific standards, which JOD supports by offering a broad variety of formats to express such ideas as well as the flexibility to seize novel opportunities; and we care about supporting and growing a strong research community that spans junior and senior scholars, novices and experts, as well as theorists and practitioners. If organization design is concerned with understanding how organizations work and how they can work better, we want JOD to be the best-possible platform for students of organizations to push the boundaries of this knowledge. We hope you will join us on this endeavor!

Publisher's Note Springer Nature remains neutral with regard to jurisdictional claims in published maps and institutional affiliations. 